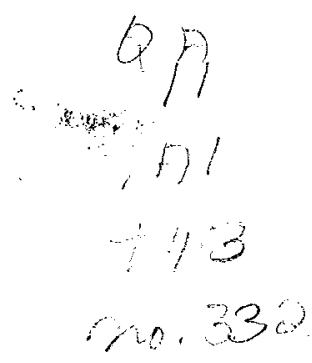

\title{
A MOLECULAR MECHANICS SIMULATION OF CRACKS AND FRACTURES IN A SHEET OF ICE \\ Donald Greenspan
}

TR \# 332

July, 1998

(817) 272-3261

FAX (817) 272-5802 


\title{
A MOLECULAR MECHANICS SIMULATION OF CRACKS AND FRACTURES IN A SHEET OF ICE
}

\author{
Donald Greenspan \\ Mathematics Department \\ University of Texas at Arlington
}

\section{Abstract}

Rectangular, two dimensional sheets of ice molecules are both stressed and compressed. Computer examples compare dynamical responses when the plate has a slot or does not have a slot. The mechanisms for both crack and fracture development are clearly delineated on the molecular level.

1. Introduction. The study of crack and fracture development is of interest in engineering, science, and mathematics (see, e.g., refs. [1]-[12] and the numerous references contained therein). Stress and compression forces, in particular, have received wide attention both experimentally and theoretically. Theoretically, emphasis has been from a continuum point of view.

In this paper we study stress and compression effects theoretically but from a molecular mechanics point of view. In particular, attention will be directed to the reaction dynamics of a thin, two dimensional sheet of ice. The simulation of compression will be limited by an assumption of nonbuckling.

Throughout, cgs units are used.

2. Basic Considerations. A 6-12 Lennard-Jones potential for a pair of ice molecules $P_{i}, P_{j}$ which are $r_{i j} \AA$ apart is

$$
\phi\left(r_{i j}\right)=(1.9646) 10^{-13}\left[\left(\frac{2.725}{r_{i j}}\right)^{12}-\left(\frac{2.725}{r_{i j}}\right)^{6}\right] \operatorname{erg}\left(\frac{\mathrm{gr} \mathrm{cm}^{2}}{\mathrm{sec}^{2}}\right) .
$$

From (2.1), the force $\vec{F}_{i j}$ exerted on $P_{i}$ by $P_{j}$ is

$$
\vec{F}_{i j}=(1.9646) 10^{-5}\left[\frac{12(2.725)^{12}}{r_{i j}^{13}}-\frac{6(2.725)^{6}}{r_{i j}^{7}}\right] \frac{\vec{r}_{j i}}{r_{i j}} \text { dynes }\left(\frac{g \mathrm{~cm}}{\mathrm{sec}^{2}}\right) .
$$

Since the mass $m$ of a water molecule is $(30.103) 10^{-24} \mathrm{gr}$, equation (2.2) can be simplified to

$$
\vec{a}_{i}=(160.33) 10^{19}\left[\frac{818.90}{r_{i j}^{13}}-\frac{1}{r_{i j}^{7}}\right] \frac{\vec{r}_{j i}}{r_{i j}}\left(\frac{\mathrm{cm}}{\mathrm{sec}^{2}}\right) \text {. }
$$

Recasting the latter equation in $\stackrel{\circ}{p^{2}}\left(1 \mathrm{sec}=10^{12} \mathrm{ps}, 1 \mathrm{~cm}=10^{8} \stackrel{\circ}{A}\right)$ yields

$$
\vec{a}_{i}=(160330 .)\left[\frac{818.90}{r_{i j}^{13}}-\frac{1}{r_{i j}^{7}}\right] \frac{\vec{r}_{j i}}{r_{i j}}\left(\frac{\stackrel{\AA}{A}}{p s^{2}}\right) .
$$


For computational convenience, however, (2.3) will be rewritten using the time transformation $T=100 t$ as

$$
\frac{d^{2} \vec{r}_{i j}}{d T^{2}}=(16.0330)\left[\frac{818.90}{r_{i j}^{13}}-\frac{1}{r_{i j}^{7}}\right] \frac{\vec{r}_{j i}}{r_{i j}} .
$$

From equation (2.2), it follows that the magnitude $F_{i j}$ of $\vec{F}_{i j}$ is zero when $r_{i j}=3.06 \AA$ and, by differentiation of $F_{i j}$, that the elastic limit is $r_{i j}=3.39 \AA$.

Note that in a regular triangle whose edge is $3.06 \AA$, the altitude is $2.65 \AA$.

Using the latter observation, we next construct a regular triangular mosaic of 2713 points using the recursion formulas

$$
\begin{aligned}
& x(1)=-59.67, y(1)=0 \\
& x(i+1)=x(i)+3.06, y(i+1)=0, i=1,39 \\
& x(41)=-61.2, y(41)=2.65 \\
& x(i+1)=x(i)+3.06, y(i+1)=y(41), i=41,80 \\
& x(i)=x(i-81), y(i)=y(i-81)+2(2.65), i=82,2713 .
\end{aligned}
$$

At each resulting grid point we place an ice molecule. The initial data are completed by assigning each molecule a velocity $\vec{v}_{i}=\overrightarrow{0}$. The initial configuration of the system is shown in Figure 1.

The neighbors of any $P_{i}$ are taken to be those molecules which are initially 3.06 $\AA$ from $P_{i}$. The neighbors of any $P_{i}$ are defined to be the neighbors of $P_{i}$ for all time and the force on any $P_{i}$ is the sum of the forces exerted on $P_{i}$ by its neighbors.

In the examples to be described, the solution of system (2.4) will be generated numerically, in double precision, by the leap frog formulas [13]. Throughout, $\Delta T=0.0001$ and $T(k)=k \Delta T, k=0,1,2, \ldots$.

3. Stress Examples. For our first example, the ice sheet in Figure 1 is stressed as follows. At each time step the very top row is moved upward $d \stackrel{\circ}{A}$ and the very bottom

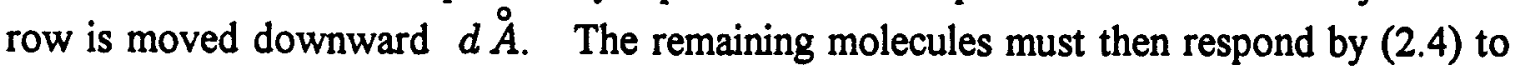
this imposed stress.

For $d=0.000001$, Figure 2 shows that the resulting force is impulsive and simply tears off the topmost and bottommost sections of the sheet.

Reducing $d$ to 0.0000004 in the above example yields the results shown in Figures 3, 4 and 5 at the respective times $T(8000000), T(15000000)$ and $T(30000000)$. The force field in Figure 6 is that for Figure 3 and reveals that the force is transmitted internally in waves. Figure 7 shows the force field for Figure 4 and indicates the directions of the cracking, which culminate in the fractures shown in Figure 5.

The present results demonstrate a strong cohesiveness of the sheet, which will be verified by the next example.

Consider now placing a slot in Figure 1 by removing the 15 molecules $P(1070+41 i), i=0,14$. The resulting sheet is shown in Figure 8. We now expect that the sheet has been weakened. Repeating the stress induced with $d=0.000001$ no longer 
results in the impulsive motion shown in Figure 2. The sheet's reaction at times $T(3000000), T(6000000), T(9000000), T(13000000)$, and $T(17000000)$ are shown in Figures $9-13$, respectively. The force fields in Figures 9 and 10 are shown in Figures 14 and 15 , which indicate how the crack patterns are developing and where the final fractures will result. Figure 16 further delineates aspects of Figure 15 by showing slash lines between those molecules of the original configuration (Figure 8) whose separation distances have exceeded the elastic limit. In this figure, slash lines which are not connected indicate a weakening of the cohesiveness of the sheet. The connected slash lines represent cracks, and these have developed from previously unconnected, but close, slash lines.

4. Compression Examples. For emphasis, we repeat that in this section we preclude the possibility of buckling.

Since we have just been dealing with a slotted plate, let us continue here by considering it first. Hence, let the initial configuration be that of Figure 8. At each time step let the uppermost row be moved downward $d \AA$ and the bottommost row be moved

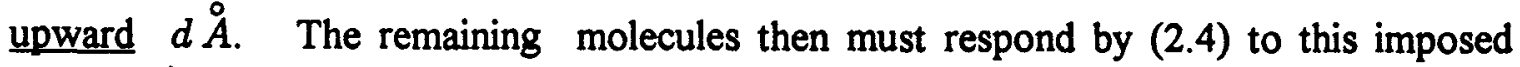
compression.

For $d=0.000002$, Figures $17-22$, which now include the force fields, show the transition to fracture at the respective times $T(1000000), T(2000000), T(3000000)$, $T(4500000), T(8000000)$, and $T(12000000)$. Figure 17 reveals again that the force field moves inward in waves. In order to predict the major areas of fracture, Figure 23 shows by slash lines where individual molecular pairs in Figure 20 at $T(4500000)$ have separated. Again, cracks are characterized by connected sets of slash lines. Thus, cracks are seen readily in the lower left, center left, center right, and upper right sections of the figure. The resulting major fractures in these areas are seen in Figure 21.

Finally, let us consider $d=0.000002$ for the plate without a slot shown in Figure 1. Then Figures 24-27 show the development of fracture at the respective times $T(2000000), T(4500000), T(8000000)$, and $T(12000000)$, and these can be compared readily with Figures 18, 20, 21, and 22. The fracture development in Figures 24-27 shows symmetry relative to a horizontal axis, a vertical axis, and relative to the center point of the plate. The fracture in Figures $17-22$ is symmetrical only relative to the center point of the plate.

5. Other Examples and Remarks. Small perturbations of the parameter choices in the examples in Sections 2 and 3 yielded entirely analogous results. Thus the examples presented are typical of all the calculations.

It is difficult to compare our results with experiments in the large. The reason for this is that, though individuals involved in molecular mechanics hope that their results extend into the large, there is no concrete proof that this is actually the case. Thus, though we can observe that the results shown in Figure 23 are similar to the experimental results of Bombolakis [12], precise comparison is difficult. We cannot construct a regular triangular grid such that the slot molecules form a $45^{\circ}$ angle with the horizontal, which was an angle considered by Bombolakis. In addition, our slot is relatively larger in the 
plate than that of Bombolakis. Yet, a large portion of the crack generated in Figure 23 is similar to that obtained by Bombolakis.

\section{REFERENCES}

1. R. Burridge (ed) Fracture Mechanics, Amer. Math. Soc., Prov. RI, 1978.

2. A. K. Chatterjee and L. Knopaff, "Fracture dynamics of fusion of two anti-plane cracks", Proc. NAS USA, 82 (1985), pp. 1869-1873.

3. E. S. C. Ching, "Dynamic stresses at a moving crack tip in a model of fracture propagation", Phys. Rev. E, 3 (1994), pp. 3382-3388.

4. A. D. Fitt, A. D. Kelly, and C. P. Please, "Crack propagation models for rock fracture in a geothermal energy reservoir", SIAM J. Appl. Math., 55 (1995), pp 1592-1608.

5. S. W. Freeman (ed), Fracture Mechanics Applied to Brittle Materials, ASTM, Phila., 1979.

6. D. Gross, "Path independent integrals and crack growth parameters in nonlinear fracture mechanics", In Nonlinear Fracture Mechanics, CISM Courses and Lectures, 314, Springer, Vienna, 1990, pp 123-141.

7. F. Kirkhof, Bruchvorge in Glasern, Verlage die Deutschen Glastetechnischen Gesellschaft, Frankfurt, 1970.

8. H. Liebowitz (ed) Fracture, an Advanced Treatise, Academic, NY, 1968.

9. Q. S. Nguyen, "Critere de propagation en rupture ductile", C. R. Acad. Sci., Paris, Sér II, 301 (1985), pp 567-570.

10. K. Ohtasuka, "Surface crack problems", Hiroshima Math. J., 16 (1986), pp 327352.

11. N. Perrone and S. N. Alturi (eds), Nonlinear and Dynamic Fracture Mechanics, ASME, NY, 1979.

12. G. C. Sih (ed) Dynamic Crack Propagation, Noordhoff, Leiden, 1973.

13. D. Greenspan, Particle Modeling, Birkhauser, Boston, 1997. 


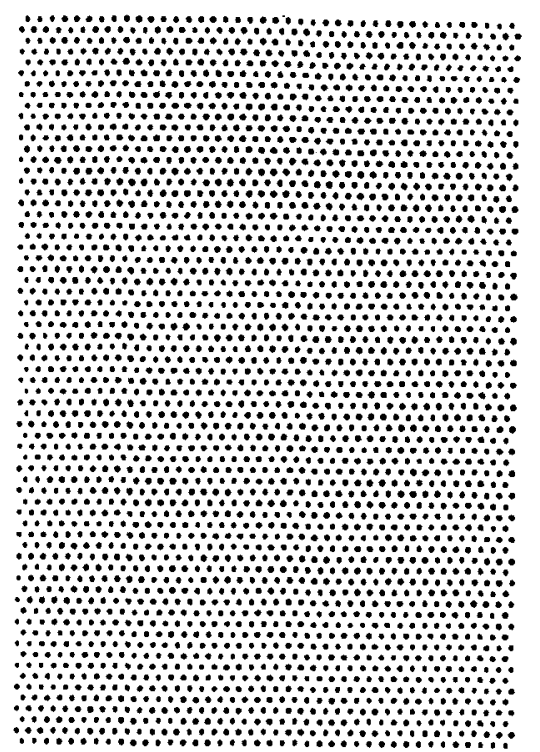

P18. I The Inttial configuration

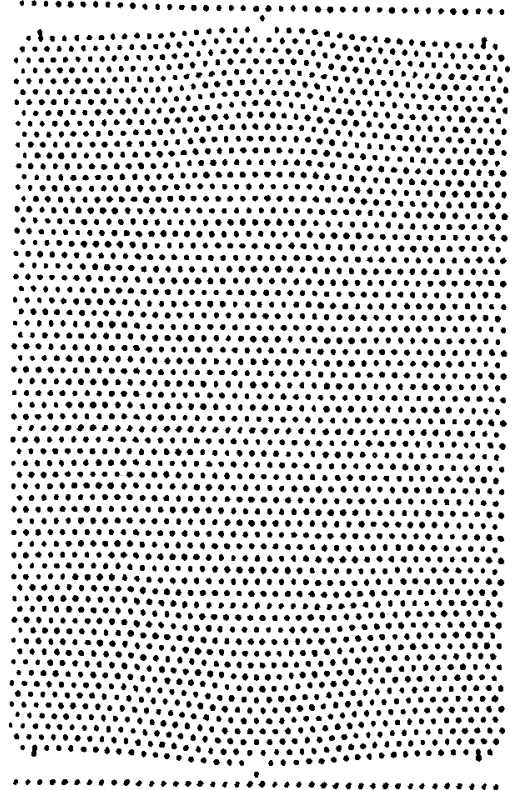

818. 2 Impula1ve reaction

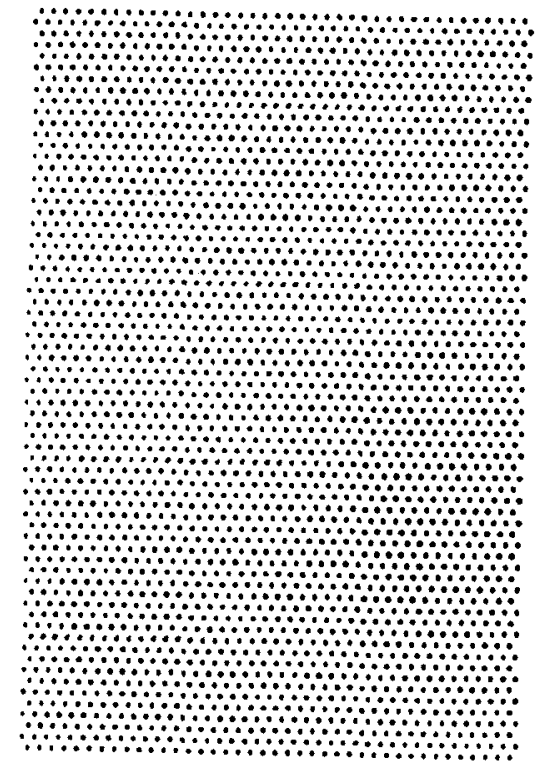

P18. $3 T=T(8000000)$

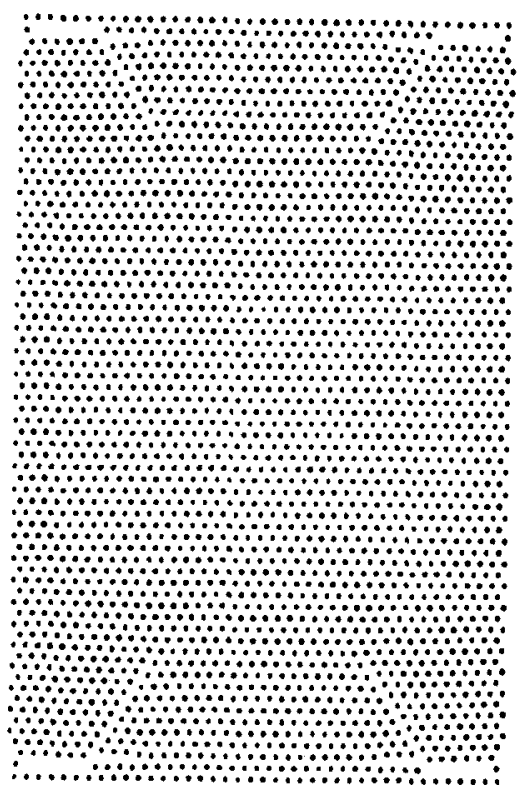

B1g. $4 T=T(15000000)$

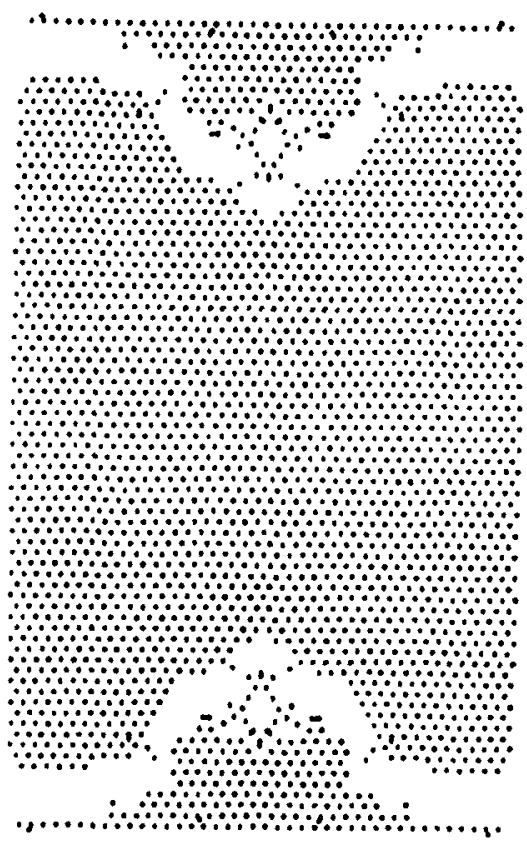

P18. $5 \mathrm{~T}=\mathrm{T}(30000000)$ 


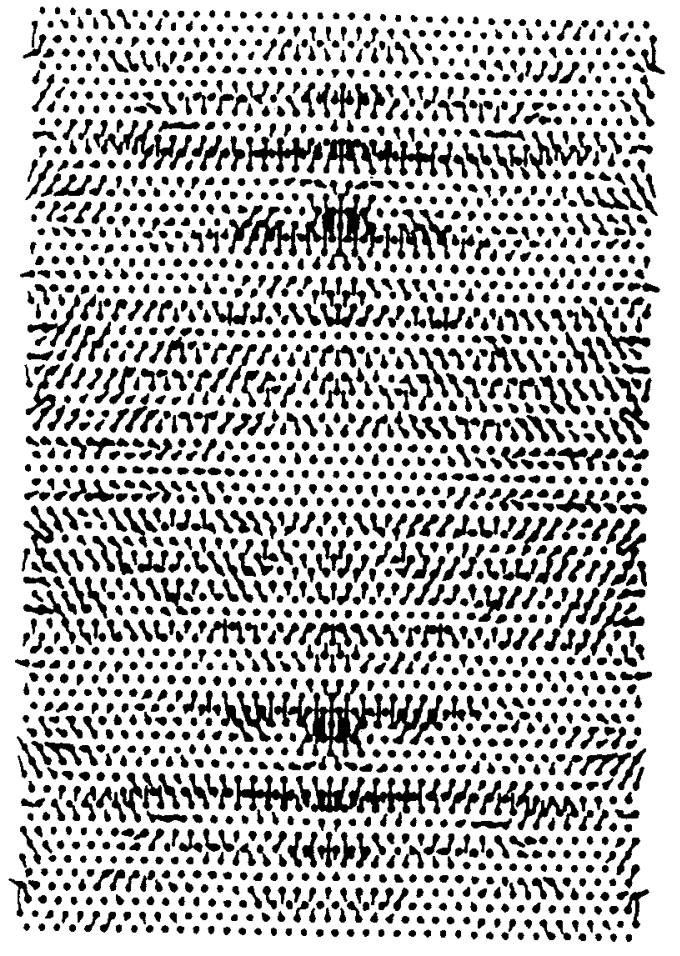

P18. 6 Porce fleld of P18. 3

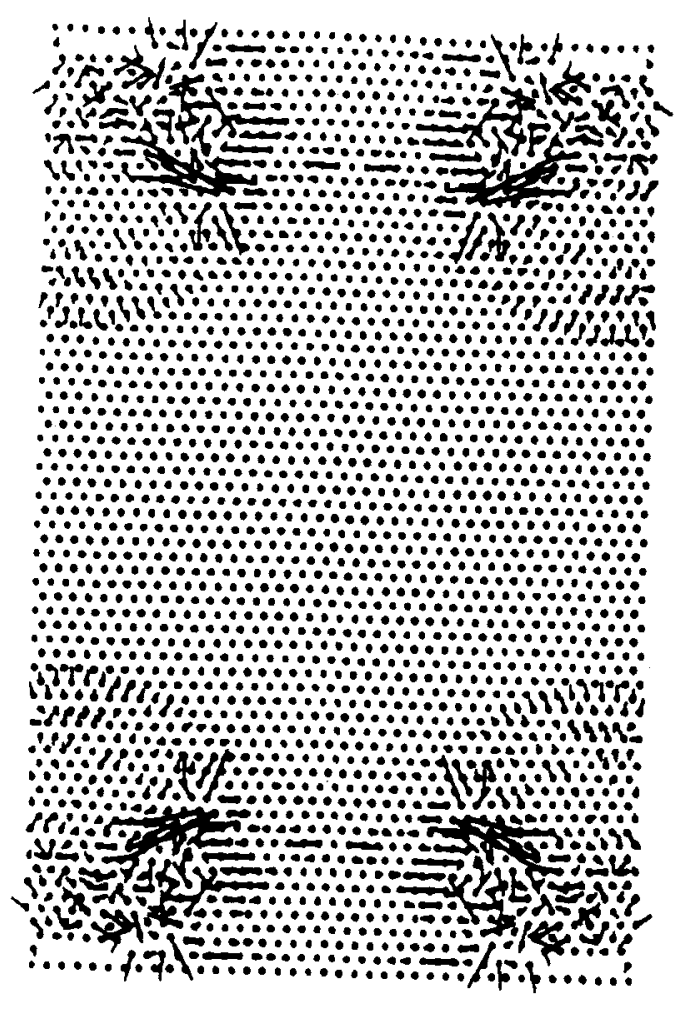

P18. 7 Porce fleld of 118.4

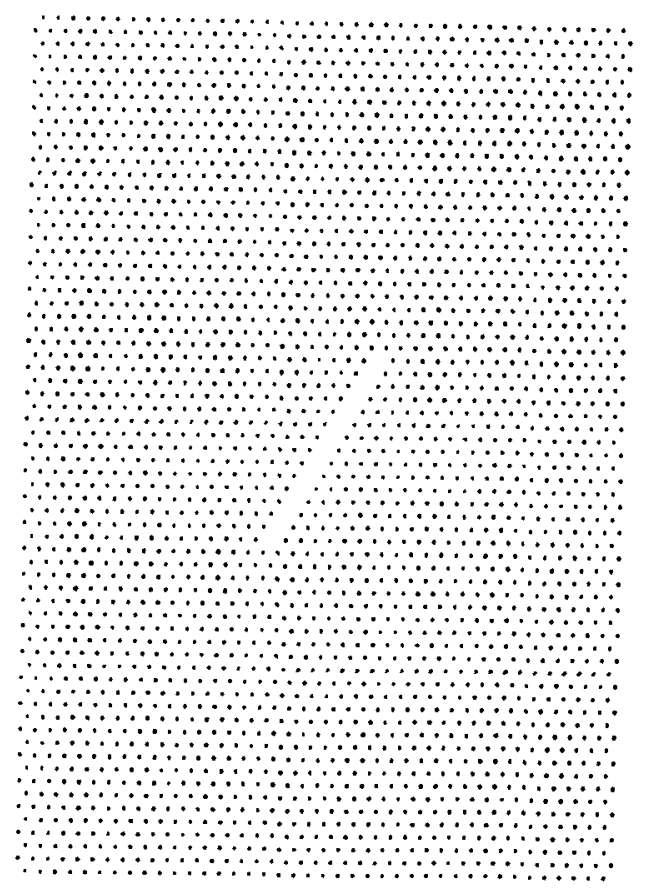

18. $8 \times 60^{\circ}$ slot 


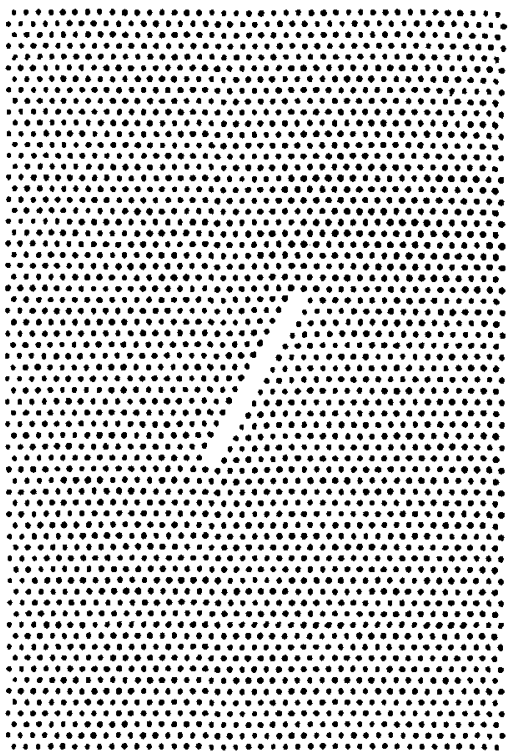

118. $9 \operatorname{Ir}(3000000)$

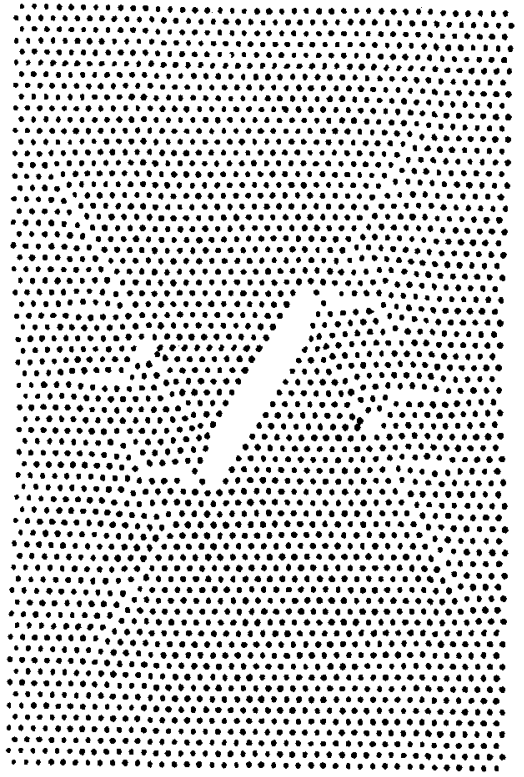

118. $10 T=T(6000000)$

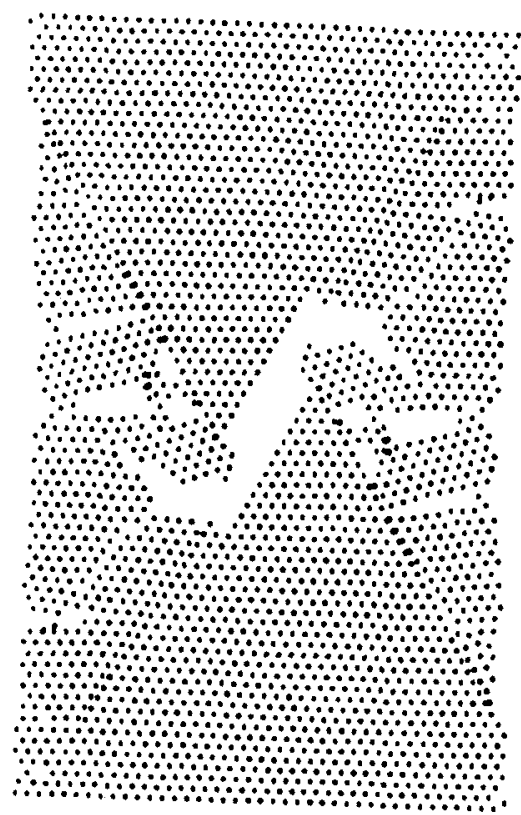

18. 11 T-T (900000n)

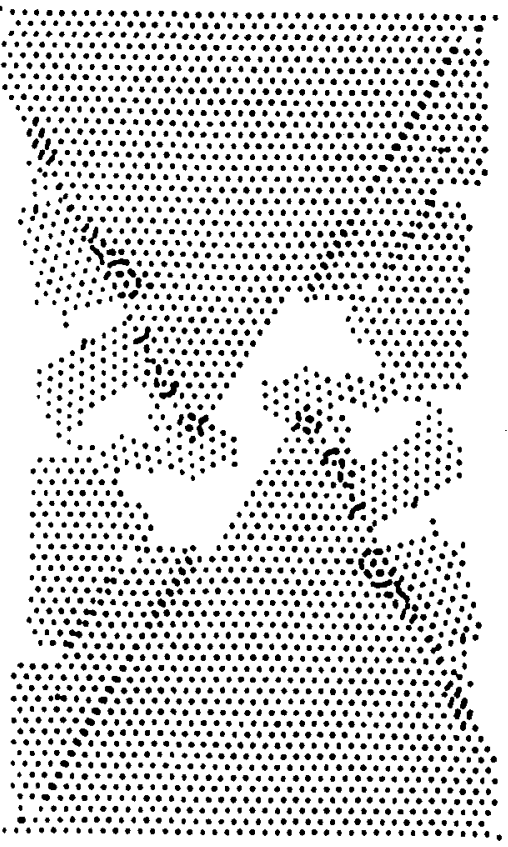

P18. $12 T=T(13000000)$

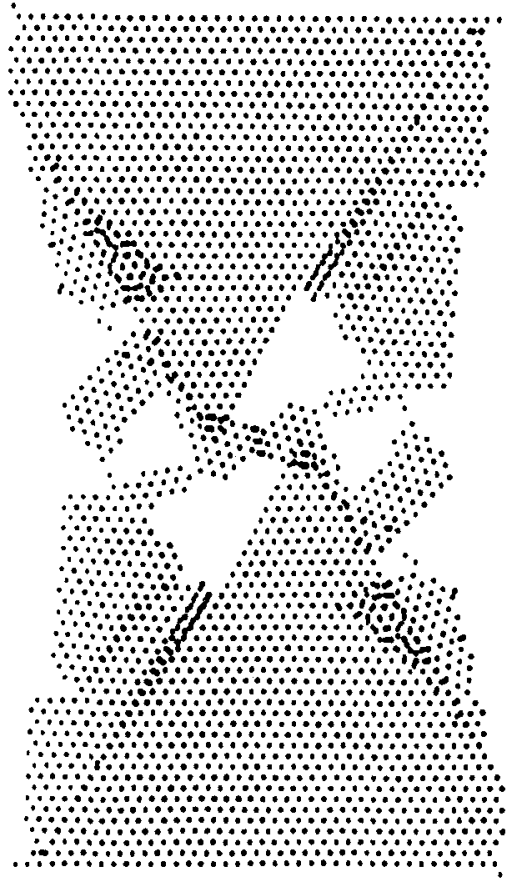

818. $13 T=T(17000000)$ 


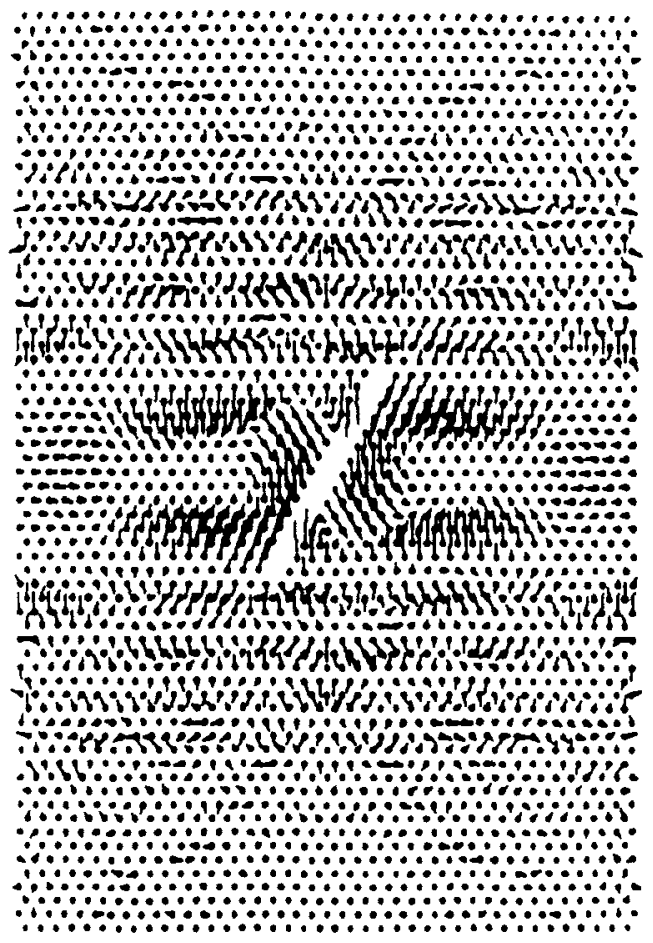

P18. 14 Porce fleld for P1g. 9

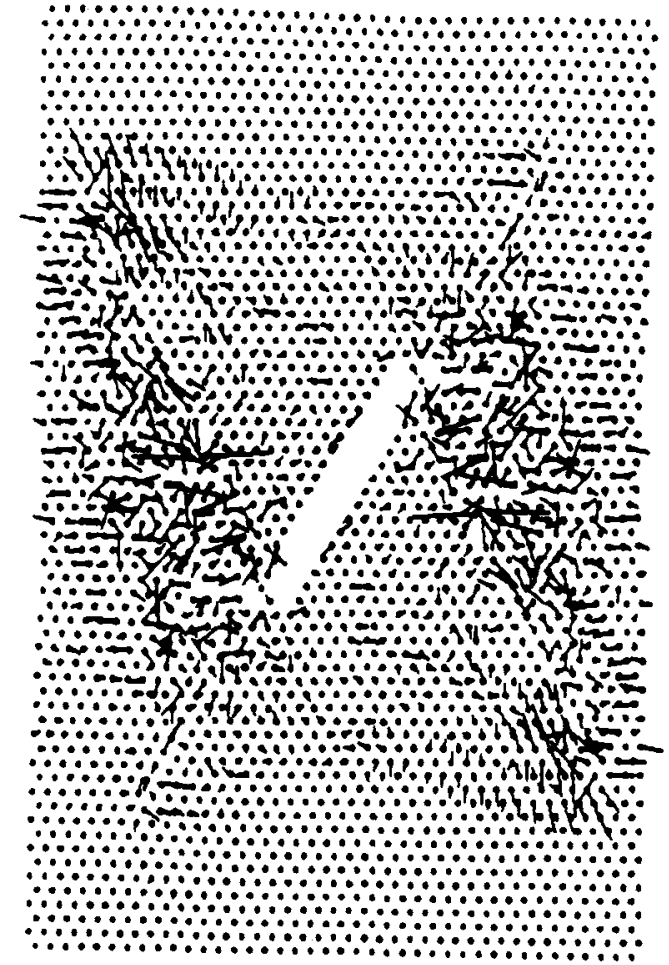

P18. 15 Porce fleld for F1g. 10 


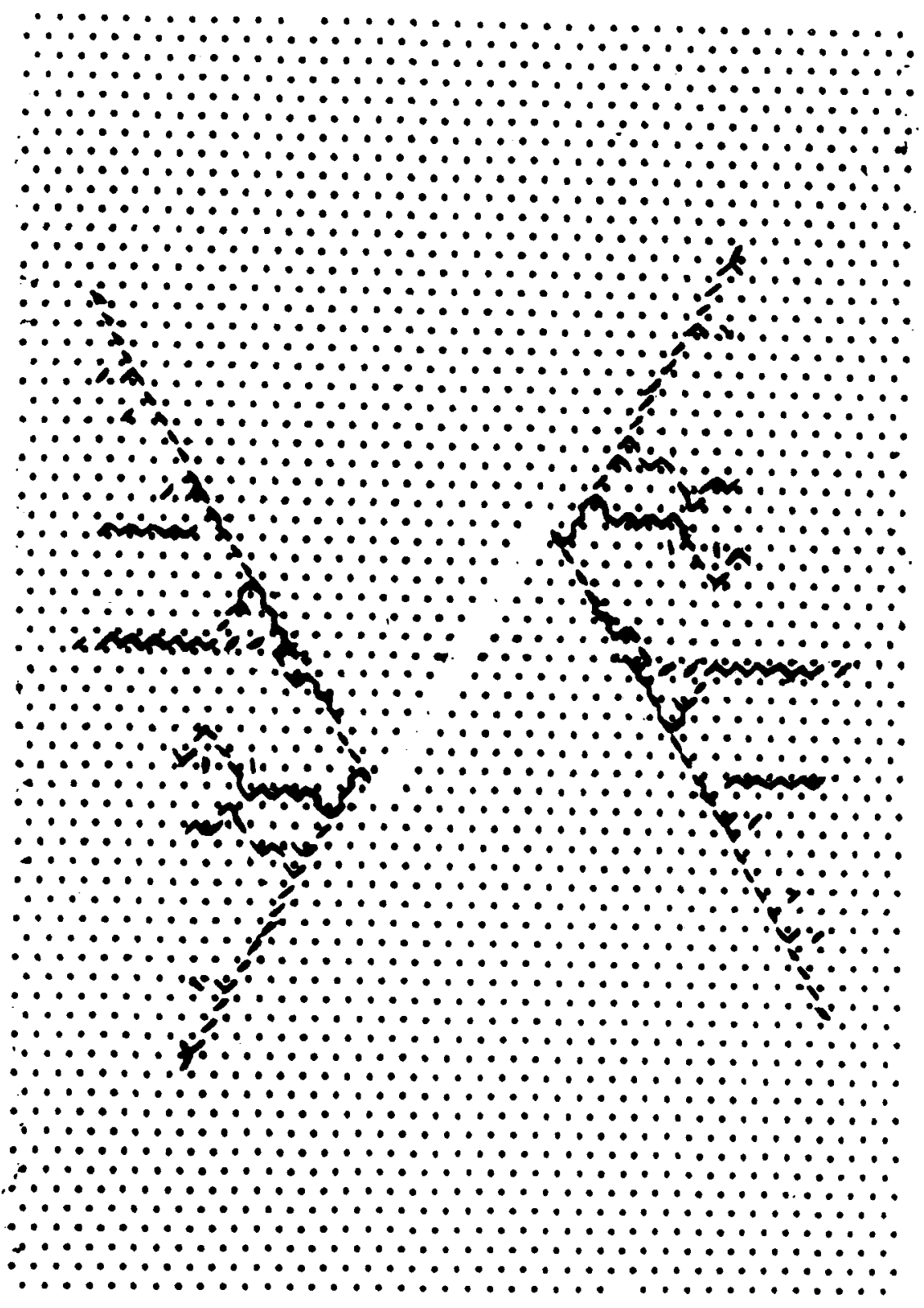

P18. I6 Molecular separat1ons and cracke 1n P1g. 15 


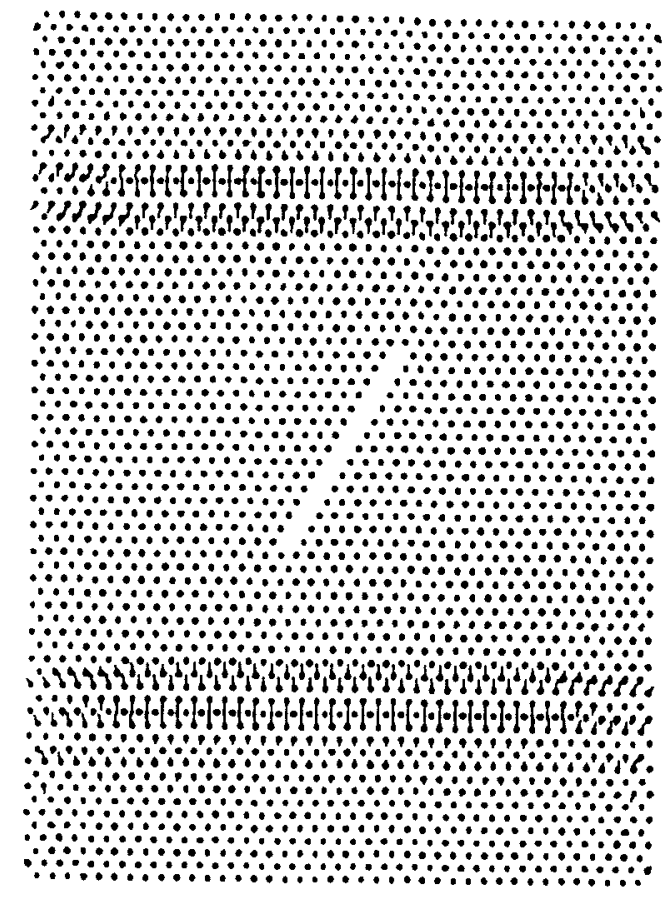

F18. $17 T=T(1000000)$

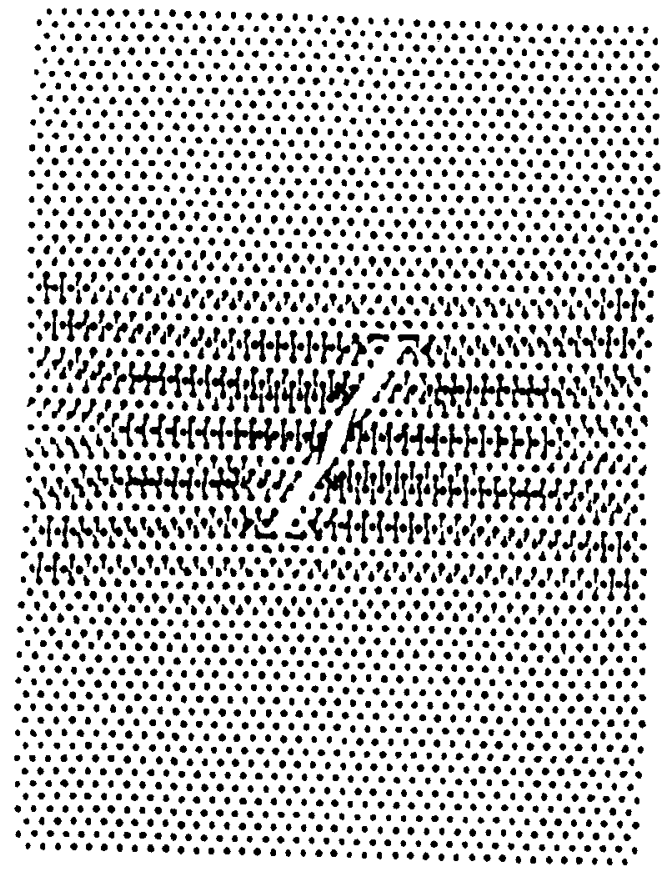

P18. $18 T=T(2000000)$

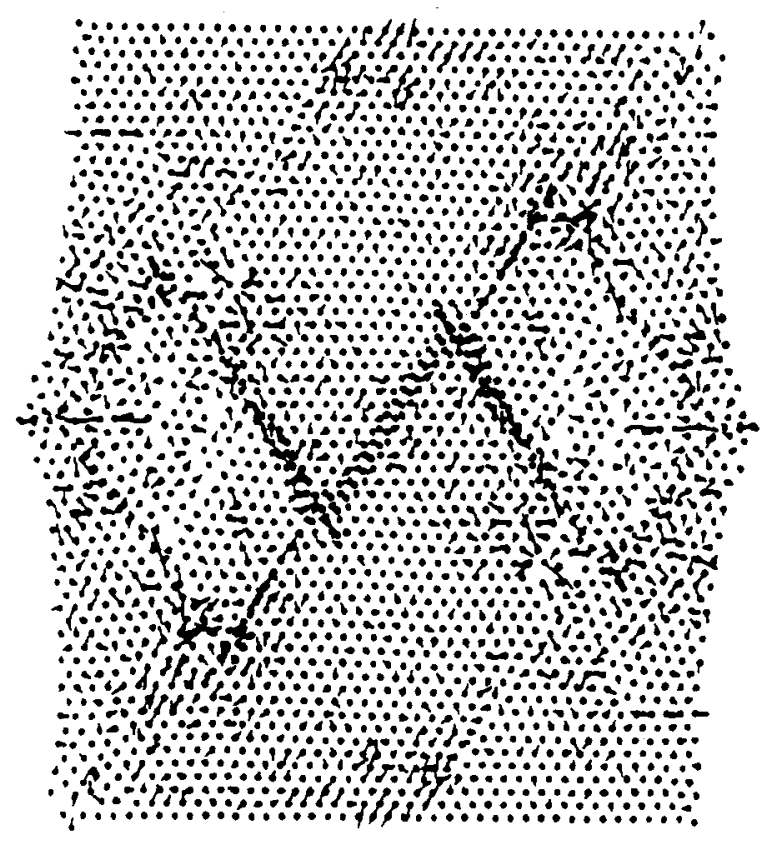

F1g. $20 \mathrm{~T}=\mathrm{T}(4500000)$ 


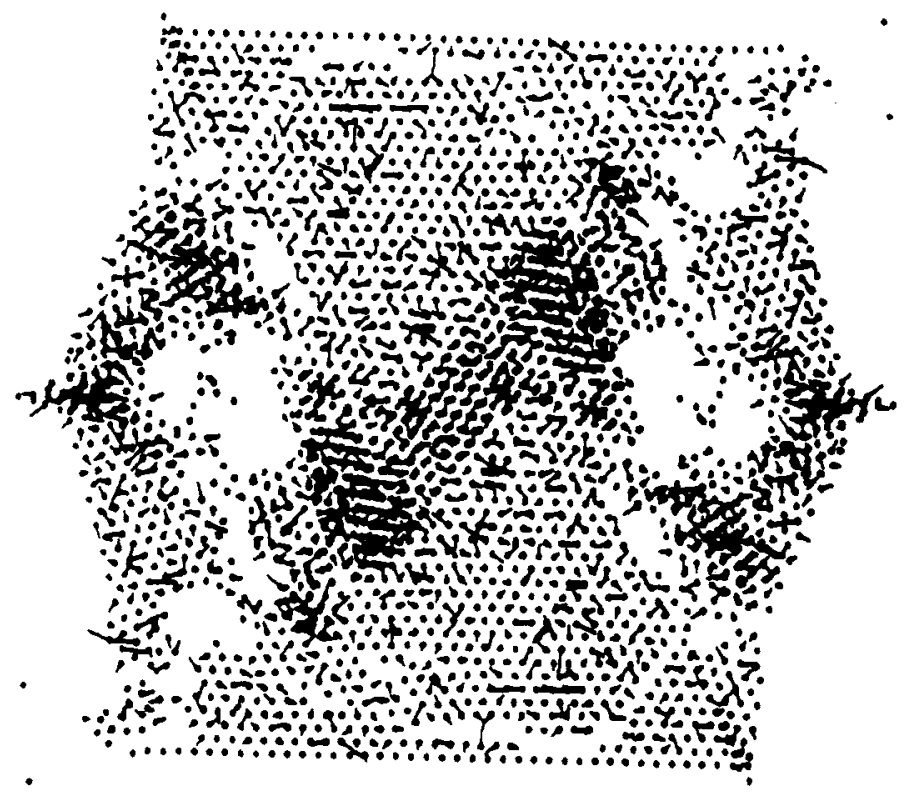

Fig. $21 \mathrm{~T}=\mathrm{T}(8000000)$

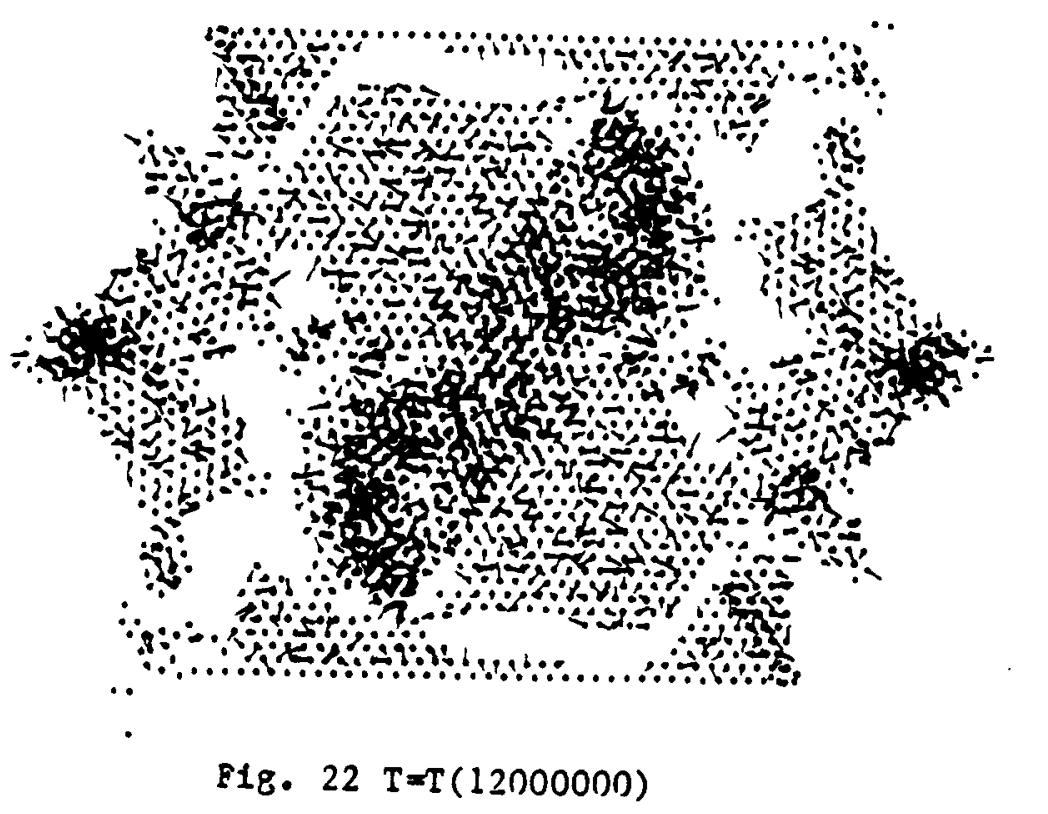




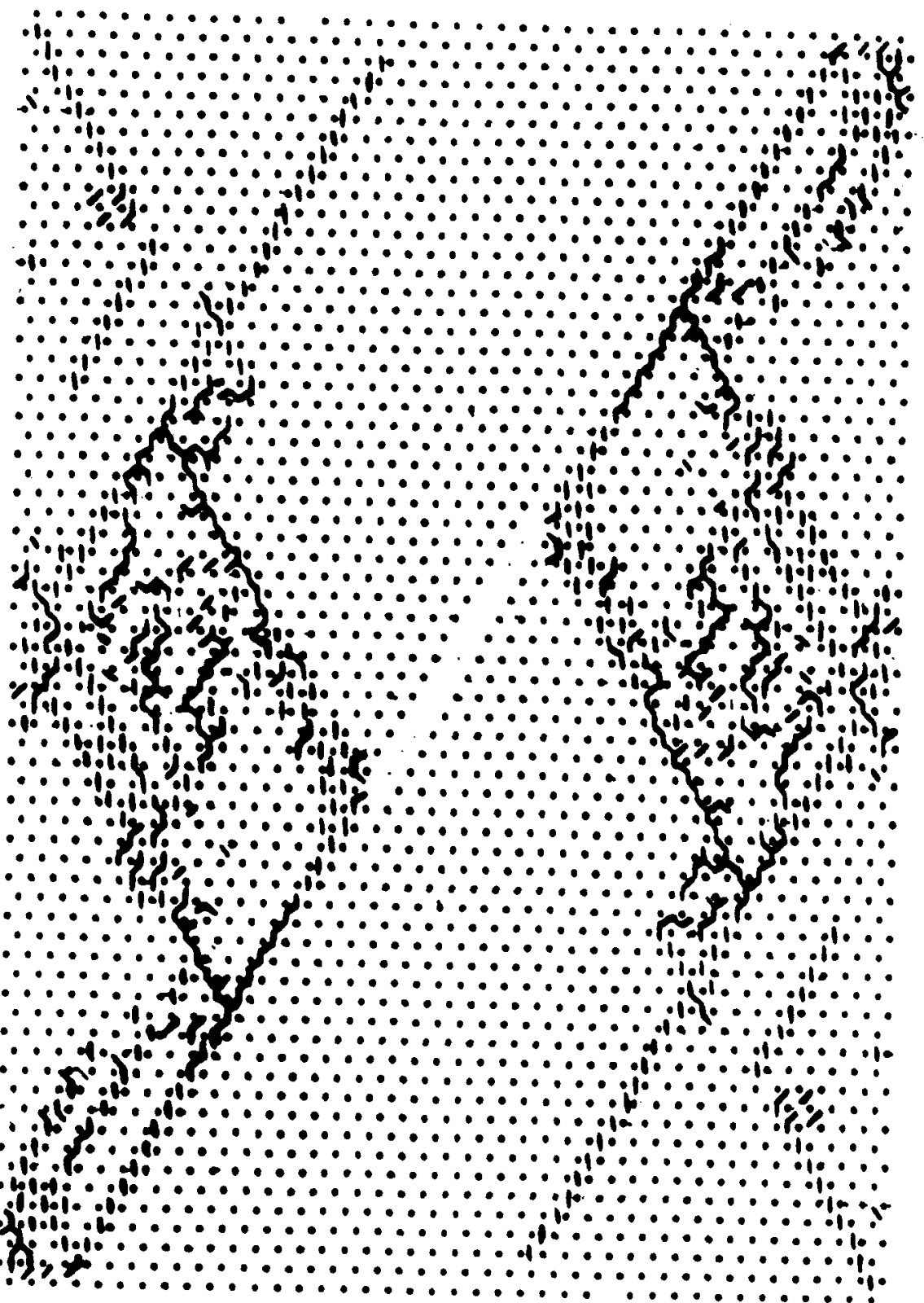

P1g. 23 Molecular separations and cracks in Ifg. 20 


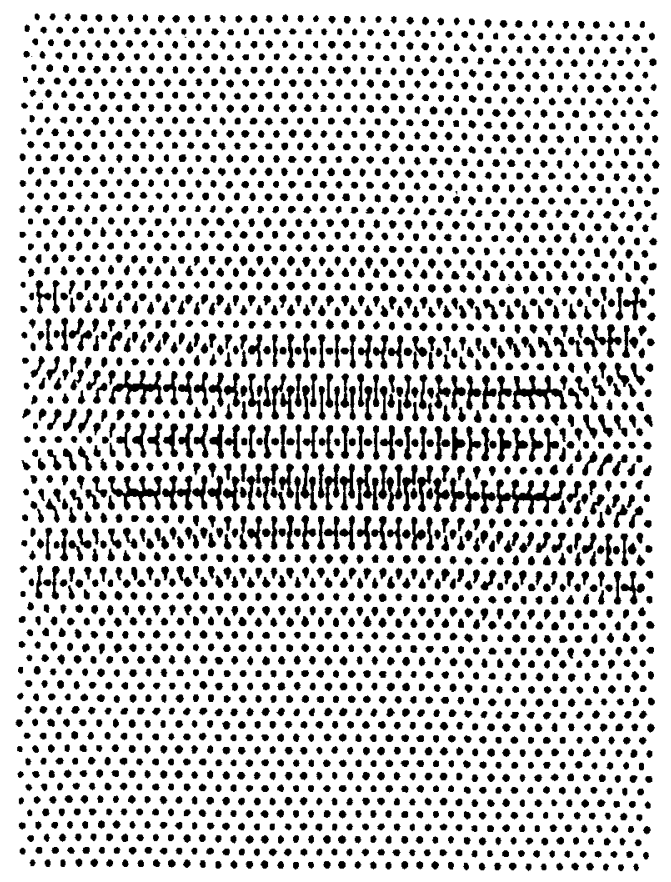

P18. $24 T=T(2000000)$

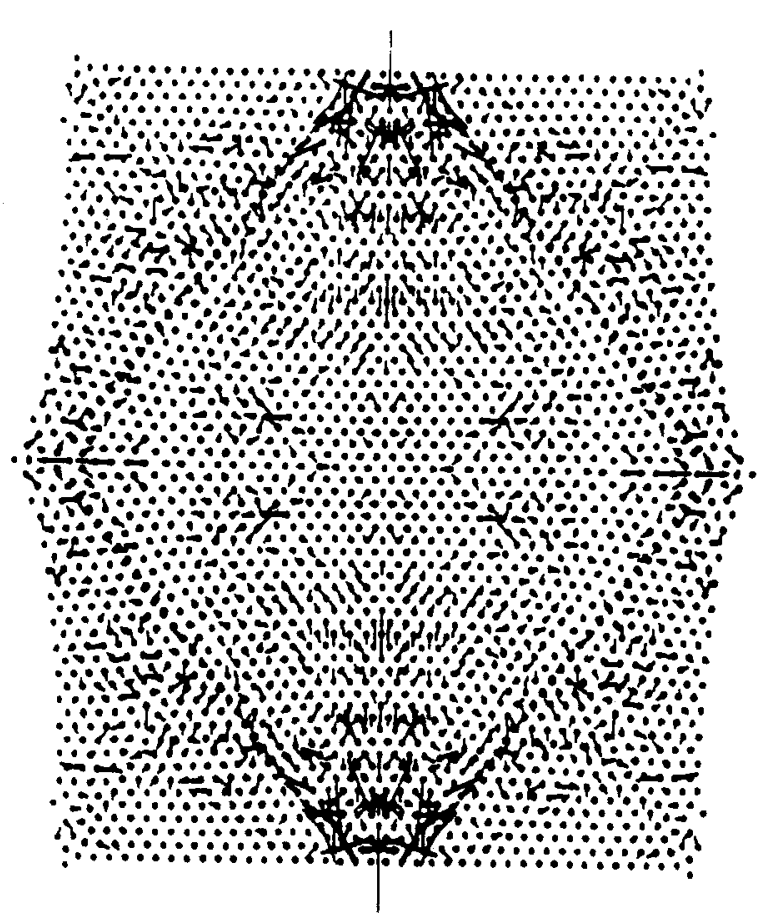

F1g. $25 T=T(4500000)$

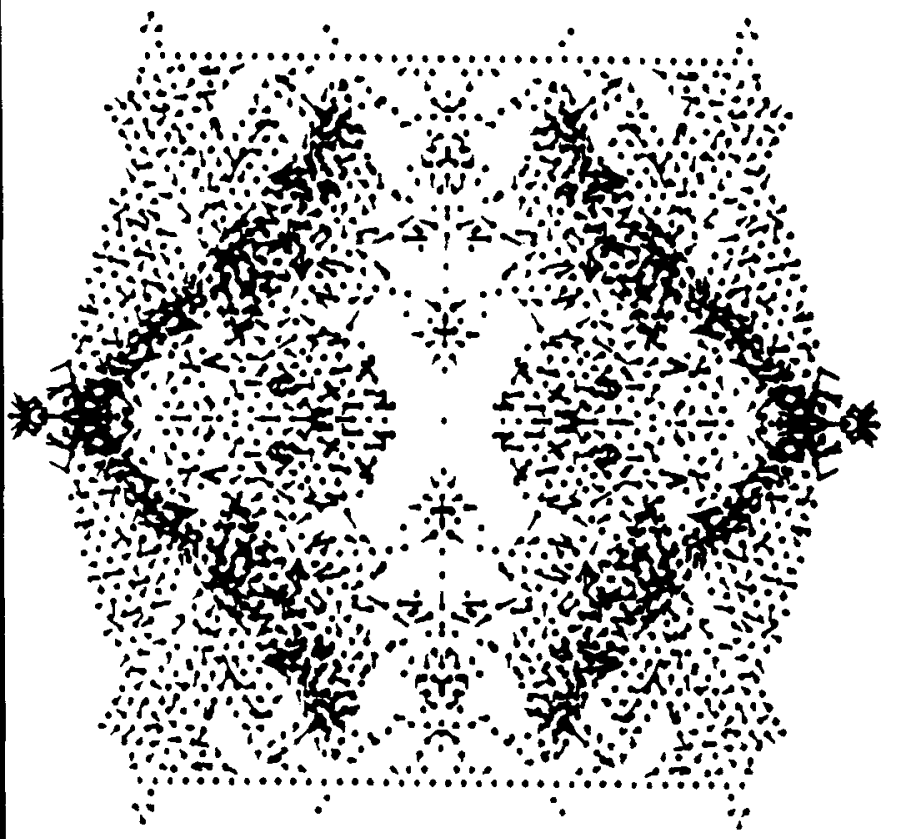

81g. $26 \mathrm{~T}=\mathrm{T}(8000000)$

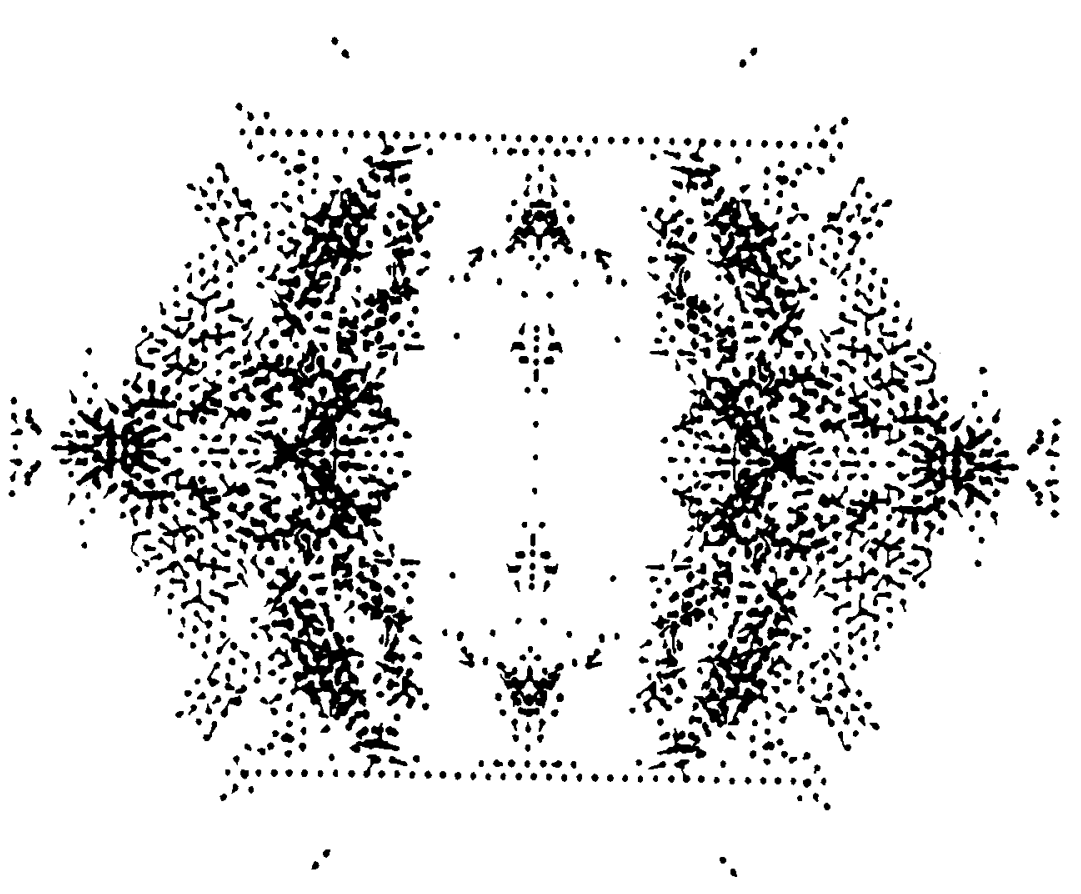

F1g. $27 \mathrm{~T}=\mathrm{T}(12000000)$ 